\title{
Closed Posterior Dislocation of Ankle without Associated Fractures: A Case Report
}

\author{
Awkash Kumar ${ }^{1}$, Sandeep K Yadav²
}

\begin{abstract}
Ankle joint is an inherently stable joint well supported with ligament and bones configuration. Ankle dislocation without fracture is a rare entity needs to be managed urgently in the form of immobilization in below knee cast for appropriate time period. Rehabilitation protocol is to be followed in order achieve targeted range of motion. Complication associated with ankle dislocation needs to be kept in mind while managing these kinds of condition.

Keywords: Ankle joint, Dislocation, Immobilization.

Journal of Foot and Ankle Surgery (Asia Pacific) (2021): 10.5005/jp-journals-10040-1135
\end{abstract}

\section{INTRODUCTION}

Ankle joint is an inherently stable joint, owing to the anatomy of the mortise and presence of strong ankle ligaments, whose strength exceeds that of bone, thus causing malleolar fracture in the event of injury. ${ }^{1}$ Ankle fractures without dislocation occur in about $80 \%$ of the cases. ${ }^{2}$ Ankle dislocation without an associated fracture, described often as pure ankle dislocation is a rare lesion, accounting for $<0.065 \%$ of injuries around ankle. ${ }^{3}$ Ligamentous hyperlaxity, history of previous ankle sprains, medial malleolar hypoplasia, and talar undercoverage are considered risk factors for ankle dislocation. ${ }^{4-6}$ We present a case of posterior ankle dislocation without associated fracture following road-traffic accident in a young healthy adult.

\section{Case Description}

A 28-year-old man presented to the emergency room with an acutely painful and grossly deformed right ankle following a roadtraffic accident. The patient stated he landed on his right foot in plantar flexion and inversion. He reported pain in the ankle but denied paresthesia or loss of power distal to the injury. There were no neurovascular, or other injuries. Examination of other joints revealed no hyperlaxity. Patient had no history of pain, recurrent sprains, or prior instability in the affected ankle.

Physical examination revealed an inversion and internal rotation deformity of the ankle with prominent lateral malleolus. His foot was neurovascular intact with brisk capillary refill and without any sensory loss. Radiographs of the ankle revealed posterior dislocation of the ankle without any fractures or widening of the tibiofibular syndesmosis.

Closed reduction of ankle was performed under light sedation with IV ketamine. Reduction was achieved after flexing the knee to $90^{\circ}$, with a distal-directed force, the foot was externally rotated and anteriorly translated and posteriorly directed counter pressure on the tibia was applied. Patient reported immediate relief of pain following the reduction. The ankle was not stressed after reduction. The ankle was immobilized in a short leg cast in neutral position for 6 weeks; the patient was allowed to ambulate using crutches
${ }^{1}$ Department of Orthopedics, Shalby Hospital, Ahmedabad, Gujarat, India

${ }^{2}$ Department of Orthopedics, Trauma and Emergency, All India Institute of Medical Sciences, Raipur, Chhattisgarh, India

Corresponding Author: Sandeep KYadav, Department of Orthopedics, Trauma and Emergency, All India Institute of Medical Sciences, Raipur, Chhattisgarh, India, Phone: +918238034069, e-mail: sandy22987@ gmail.com

How to cite this article: Kumar A, Yadav SK. Closed Posterior Dislocation of Ankle without Associated Fractures: A Case Report. J Foot Ankle Surg (Asia Pacific) 2021;8(1):33-35.

Source of support: Nil

Conflict of interest: None

without bearing any weight on foot. After removal of the cast, good range of motion (ROM) of the ankle was demonstrated $\left(20^{\circ}\right.$ dorsiflexion, $30^{\circ}$ plantar flexion) and partial weight-bearing was started at 6 weeks. Full weight-bearing was permitted after 8 weeks. He resumed all normal activities by 3 months (Figs 1 to 4).
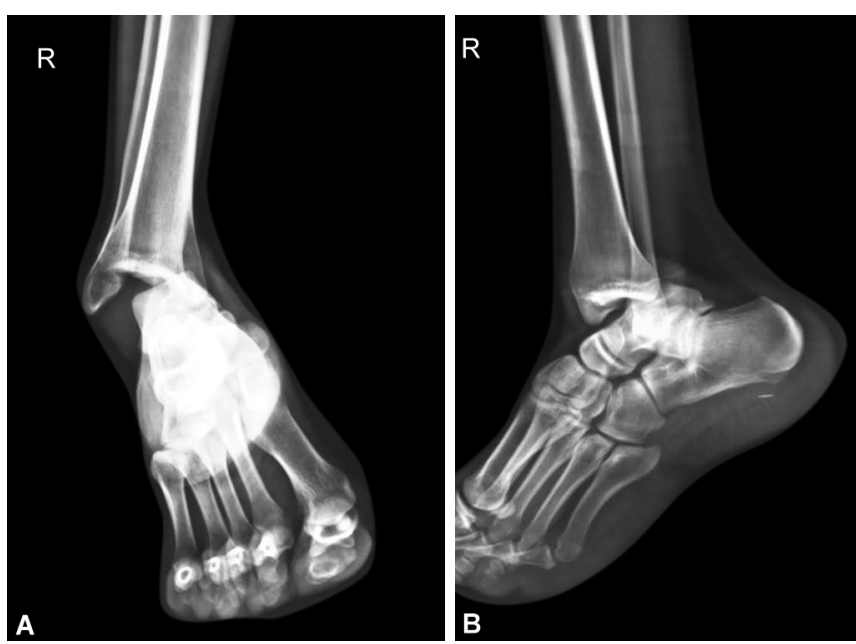

Figs $1 \mathrm{~A}$ and B: Prereduction X-ray

() The Author(s). 2021 Open Access This article is distributed under the terms of the Creative Commons Attribution 4.0International License (https://creativecommons. org/licenses/by-nc/4.0/), which permits unrestricted use, distribution, and non-commercial reproduction in any medium, provided you give appropriate credit to the original author(s) and the source, provide a link to the Creative Commons license, and indicate if changes were made. The Creative Commons Public Domain Dedication waiver (http://creativecommons.org/publicdomain/zero/1.0/) applies to the data made available in this article, unless otherwise stated. 


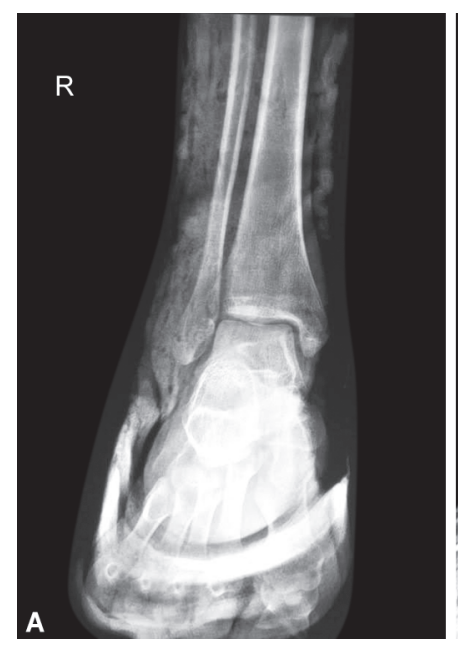

Figs 2A and B: Postreduction X-ray

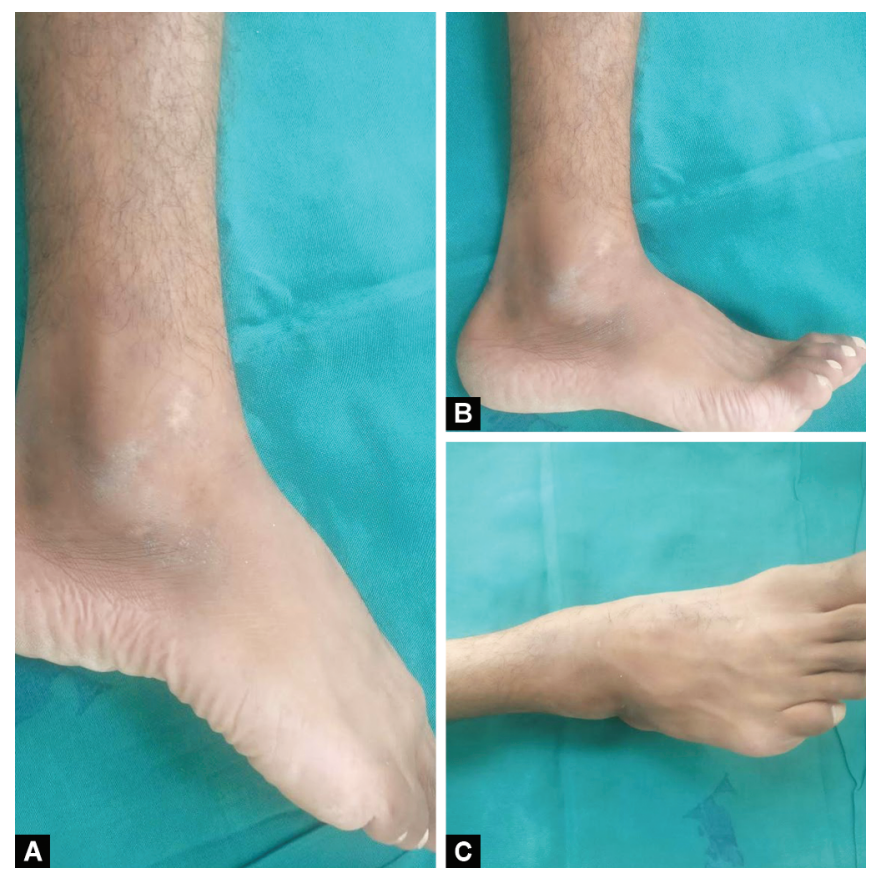

Figs 4A to C: Clinical photograph at follow-up

\section{Discussion}

Closed ankle dislocation without fracture or syndesmotic injury is quite rare representing only 13 per 20,000 ankle injuries which can be either due to sports injury or motor vehicle accident. ${ }^{4}$ This rarity is attributed to stronger ligamentous structures around the stable ankle mortise and relatively weaker malleoli. Hence, there is a chance of malleoli fracture before ligaments getting torn. ${ }^{7}$ The congruency of talus in ankle mortise along with the medial side ligaments including the deep and the superficial deltoid ligaments and the lateral ones include the anterior talofibular ligament (ATFL), calcaneofibular ligament (CFL), and posterior talofibular ligament (PTFL) which enforces the mediolateral stability of the ankle joint. The anterior to posterior stability of ankle is provided by the joint capsule and the muscles around the joint. ${ }^{8}$ When the foot is kept in maximal plantarflexion, the talus congruency in ankle mortise
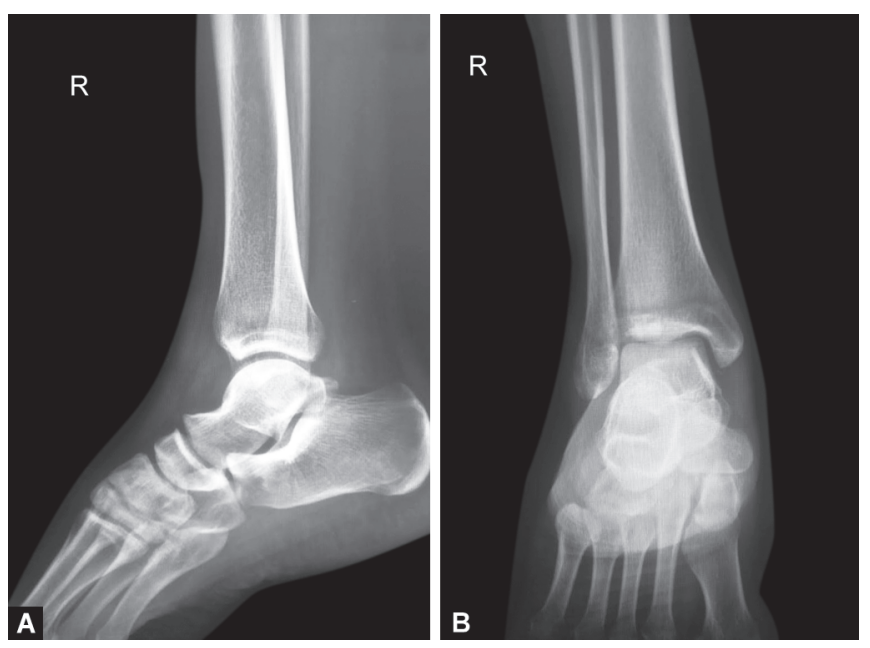

Figs $3 A$ and B: Six-week follow-up X-ray

decreases and the superadded inversion force can cause the pure dislocation of ankle joint especially in the posteromedial direction which accounts for $46 \%$ of such injuries. Most encountered predisposing factors for pure ankle dislocation includes generalized ligamentous laxity, hypoplasia of the medial malleolus, peroneal muscles weakness, and uncommonly recurrent ankle sprain. ${ }^{9}$

Early complications like vascular and neurological injuries following ankle dislocations are seen uncommonly in closed injury. Rarely joint stiffness, joint instability, degenerative arthritis, etc., can be expected as late complications.

Closed reduction and conscious sedation and immobilization of ankle joint by short leg cast for 6-8 weeks is the appropriate treatment method. Early reduction can benefit the patient by reducing the swelling formation and relieving pressure on neurovascular bundles. However, second-line radiological investigation, such as computed tomography (CT) scan, can be recommended to rule out any osteochondral fragments in the joint.

Although maximum patients recover completely, few may show up later with residual pain, residual instability, arthritic changes, and very rarely avascular necrosis (AVN) of the talus, their evaluation by magnetic resonance imaging (MRI) is recommended.

In summary, pure closed ankle dislocation is a rare injury which can be treated by closed reduction and immobilization followed by physiotherapy. However, one should keep a close watch on anticipated complications and their timely evaluation.

\section{References}

1. D'Anca AF. Lateral rotatory dislocation of the ankle without fracture. J Bone Joint Surg 1970;52(8):1643-1646. DOI: 10.2106/00004623197052080-00014.

2. Sloane D, Coutts MB. Traumatic dislocation of the ankle without fracture-a case report. J Bone Joint Surg 1937;19:1110-1112.

3. Wight L, Owen D, Goldbloom D, et al. Pureankle dislocation: a systematic review of the literature and estimation of incidence. Injury 2017;48(10):2027-2034. DOI: 10.1016/j.injury.2017.08.011.

4. Moehring HD, Tan RT, Marder RA, et al. Ankle dislocation. J Orthop Trauma 1994;8(2):167-172. DOI: 10.1097/00005131-19940400000015.

5. Toohey JS, Worsing RA. A long-term follow-up study of tibiotalar dislocations without associated fractures. Clin Orthop Relat Res 1989(239):207-210. DOI: 10.1097/00003086-198902000-00023. https://www.ncbi.nlm.nih.gov/pubmed/2912624. 
6. Thangarajah T, Giotakis N, Matovu E. Bilateral ankle dislocation without malleolar fracture. J Foot Ankle Surg 2008;47(5):441-446. DOI: 10.1053/j.jfas.2008.05.004.

7. Orapin J, Sangasoongsong P, Thamyongkit S, et al. Case report: closed posteromedial dislocation of the ankle without medial malleolar fracture. Clin Res Foot Ankle 2016;4:197. DOI: 10.4172/2329910X.1000197.
8. Mubark I, Anwar S, Hayward K. Closed posterior ankle dislocation without associated fractures: a case report. J Surg Case Rep 2017;2017(8):rjx165. DOI: 10.1093/jscr/rjx165.

9. Devan I, Brent G, Bruce L. Isolated posterior medial ankle dislocation with associated os trigonum dislocation after low-energy mechanism. Case Reports in Orthopedics 2020;2020:5026058. DOI: 10.1155/2020/5026058. 